\title{
OBESIDADE E ORGANIZAÇÕES: UMA AGENDA DE PESQUISA ${ }^{1}$
}

\author{
Cintia Rodrigues de O. Medeiros $^{2}$ \\ Miriam de Castro Possas ${ }^{3}$ \\ Valdir Machado Valadão Júnior ${ }^{4}$
}

http://dx.doi.org/10.1590/1413-2311.173.63838

\section{RESUMO}

A obesidade não tem recebido a atenção merecida no campo de estudos sobre organizações e gestão. Existe uma lacuna nos estudos sobre diversidade e trabalho quanto à obesidade como categoria a ser considerada parte da vida organizacional, ainda que essa seja apontada, mundialmente, como um desafio social e de saúde pública. Neste artigo de natureza teórica, discutimos aspectos relevantes sobre o tema com o objetivo de oferecer uma agenda para estimular conversações de pesquisa sobre a obesidade na análise organizacional. Apresentamos nossa análise estruturada em dois pontos: (1) obesidade e política do corpo; e (2) significados simbólicos da obesidade nas práticas organizacionais. Esses dois pontos são articulados com a visão instrumental e a visão crítica.

Palavras-chave: Obesidade. Organizações. Pesquisas.

\section{OBESITY AND ORGANIZATIONS: A RESEARCH AGENDA}

\begin{abstract}
Obesity has not received the attention it deserves in the study of organizations and management. There is a gap in the study of diversity and work regarding obesity as a

\footnotetext{
${ }^{1}$ Recebido em 07/04/2016, aprovado em 18/07/2017.

${ }^{2}$ Universidade Federal de Uberlândia (Brasil) - cintia@ufu.br

${ }^{3}$ Universidade Federal de Uberlândia (Brasil) - $\underline{\text { miriam.possas@gmail.com }}$

${ }^{4}$ Universidade Federal de Uberlândia (Brasil) - valdirjr@ufu.br
} 
category of organizational life, although the obesity is, worldwide, a social and public health challenge. In this theoretical paper, we discuss relevant aspects of this subject with the aim of providing an agenda to stimulate researches on obesity in organizational analysis. We present our analysis on two points: (1) obesity and body politics; and (2) the symbolic meanings of obesity in organizational practices. We articulated these two points with the instrumental view and critical view.

Keywords: Obesity. Organizations. Research.

\section{RESUMEN}

\section{OBESIDAD Y GESTIÓN: UNA AGENDA DE INVESTIGACIÓN}

La obesidad no ha recibido la atención que merece en el campo de los estudios sobre las organizaciones y la gestión. Existe una brecha en los estudios sobre la diversidad y el trabajo con relación a la obesidad como categoría para ser considerado como parte de la vida de la organización, aunque sea considerada, en todo el mundo, como un reto social y de salud pública. En este artículo teórico, se discuten aspectos relevantes del tema con el objetivo de ofrecer una agenda de investigación para estimular conversaciones acerca de la obesidad en el análisis de la organización. Presentamos nuestro análisis estructurado en dos puntos: (1) obesidad y política del cuerpo; y (2) significados simbólicos de la obesidad en las prácticas organizacionales. Estos dos puntos se articulan con la concepción instrumental y la concepción crítica.

Palabras-clave: Obesidad. Organización. Investigación.

\section{INTRODUÇÃO}

A obesidade tem sido foco de interesse, de forma mais proeminente, em pesquisas nas áreas da saúde, psicologia, esporte e sociologia. Nas pesquisas no campo das organizações, a despeito do crescimento das discussões sobre diversidade, a obesidade tem sido inexpressiva como objeto de estudo (LEVAY, 2013), principalmente, no âmbito nacional (LEVRINI, 2016). Essa quase ausência de estudos pode ser constatada nos 
resultados das buscas em eventos e periódicos na área de Administração. Considerando o período de 1990 a março de 2016, na base de dados do Scientific Periodicals Eletronic Library (SPELL), que reúne os periódicos com Qualis/CAPES, a busca pelo termo obesidade não resultou em nenhum artigo; nos eventos da Associação Nacional de Cursos de PósGraduação em Administração (ANPAD), dois artigos resultaram quando se fez a busca com o mesmo termo. Em outros eventos da área, como os Seminários de Administração (SEMEAD), o resultado da busca não apontou nenhuma publicação. Isso denota uma lacuna quando se analisam os resultados de pesquisas que apontam para a obesidade como uma questão de saúde pública em todo o mundo, cujas consequências ultrapassam os problemas de saúde física.

Conforme relatório publicado pela consultoria McKinsey (2014), quase um terço da população mundial sofre de sobrepeso ou de obesidade, o que pode acarretar em um custo mundial de dois trilhões de dólares. A World Health Organization (2014) descreveu a obesidade como uma epidemia relacionada a diversas doenças não transmissíveis, como o diabetes, câncer e doenças cardíacas, e, ainda, atribui 2,8 milhões de mortes anuais ao peso excessivo do corpo.

A obesidade tem afetado grande parte da população do mundo e, no Brasil, sua prevalência é maior nos estratos de menor renda e da população adulta do sexo feminino, sendo nesse grupo duas vezes maior do que em homens com idade superior a 40 anos (MARIANO; MONTEIRO; PAULA, 2013). Conforme as estatísticas mundiais sobre saúde, a obesidade mais do que duplicou, desde 1980. Em 2014, mais de 1,9 bilhão de adultos com 18 anos ou mais estavam acima do peso, e desses, mais de 600 milhões eram obesos, 39\% dos adultos com 18 anos ou mais estavam acima do peso, e 13\% eram obesos. A maioria da população do mundo vive em países onde o sobrepeso e a obesidade matam mais pessoas do que outras doenças (WHO, 2014).

Puhl e Brownell (2001) discutem as atitudes e comportamentos discriminatórios contra indivíduos obesos e apontam como e porque essa discriminação sistemática ocorre. Os autores documentam uma clara e consistente estigmatização e, em alguns casos, a discriminação ocorre em três áreas importantes: trabalho, educação e saúde. Segundo os autores, $28 \%$ dos professores entrevistados em um estudo disseram que tornar-se obeso é a pior coisa que pode acontecer a alguém; $24 \%$ de enfermeiros manifestaram repulsa por pacientes obesos; e no campo educacional, os resultados apontaram que os pais dão menor suporte aos filhos estudantes obesos. 
Em âmbito nacional, Kubota (2014) realizou uma pesquisa para analisar a discriminação contra estudantes obesos e muito magros, encontrando que os alunos que se autoclassificam como muito gordos são mais sujeitos a sofrer bullying frequente. Segundo o autor, a sociedade atribui a responsabilidade da obesidade aos indivíduos, associando-a com preguiça e outros atributos negativos. Paralelo a isso, os padrões de beleza ditados na sociedade contemporânea, o baixo custo de comidas processadas e ricas em substâncias que contribuem para o ganho de peso, e o estilo de vida moderno que impõe um gasto cada vez menor de calorias são fatores que contribuem para o aumento da obesidade e para sua estigmatização.

Recentemente, o Governo de São Paulo impediu a contratação de professores obesos aprovados em concurso público, alegando inaptidão para o exercício da função (FOLHA de S.PAULO, 2011). A empresa Catho (2014) divulgou os resultados de uma pesquisa que aponta para a rejeição contra pessoas obesas por parte de $69 \%$ dos profissionais. Essa rejeição é explicada pela valorização da imagem pessoal na sociedade contemporânea, uma vez que o corpo considerado ideal pelos padrões de beleza atuais é de extrema magreza (ISTO É, 2011).

Diante dessas considerações, é pertinente e necessário discutir a obesidade no âmbito das organizações. Neste artigo de natureza teórica, discutimos aspectos relevantes sobre o tema com o objetivo de oferecer uma agenda para estimular conversações de pesquisa sobre a obesidade na análise organizacional.

Inicialmente, tecemos considerações iniciais sobre a obesidade e prosseguimos estabelecendo articulações entre obesidade e organizações. Em seguida, apresentamos uma matriz norteadora para uma agenda de pesquisa sobre o tema. Ao final, apresentamos nossas considerações finais.

\section{CONSIDERAÇÕES INICIAIS SOBRE A OBESIDADE: CONCEITOS, CONTEXTO E ESTATÍSTICAS}

Definir obesidade requer uma incursão nas publicações no campo da saúde, pois trata-se de uma doença que têm se expandido dramaticamente nas últimas décadas, a ponto de autoridades da saúde pública reconhecerem-na como uma das ameaças mais perigosas para a morbidade e mortalidade. Estando associada com um conjunto de doenças crônicas, incluindo diabetes e depressão, a obesidade é frequentemente ligada ao aumento dos custos com saúde, incapacidade para o trabalho e perda de salários (MORRIS, 2007). Segundo a 
Organização Mundial de Saúde, sobrepeso e obesidade são definidos como acúmulo de gordura anormal ou excessivo que apresenta um risco para a saúde. Uma medida para definir o nível de obesidade é o índice de massa corporal (IMC), o qual é calculado pelo peso de uma pessoa (em quilogramas) dividido pelo quadrado da sua altura (em metros). Uma pessoa com um IMC de 30 ou mais é, geralmente, considerado obeso; e um IMC igual ou superior a 25 caracteriza uma pessoa com excesso de peso (WHO, 2014).

As origens e causas da obesidade não estão ainda totalmente esclarecidas, existindo certo consenso de que a interação de múltiplos fatores pode contribuir para sua ocorrência, o que lhe confere um caráter multifatorial (STUNKARD, 2000; FERREIRA; MAGALHÃES, 2006). Embora já tenham sido considerados um problema apenas em países de alta renda, o excesso de peso e a obesidade estão agora dramaticamente em ascensão em países de baixa e média renda, especialmente, em ambientes urbanos. Sobrepeso e obesidade são colocados pelos especialistas como os principais fatores de risco para uma série de doenças crônicas, incluindo diabetes, doenças cardiovasculares e câncer (WHO, 2014). A obesidade não significa apenas o risco de desenvolvimento de doenças, mas, também, significa conviver com dificuldades diárias, como comprar roupas, conseguir um emprego, além de interferir nas relações afetivas do indivíduo (MARIANO; MONTEIRO; PAULA, 2013).

No campo da saúde, a obesidade ganha interesse, principalmente, pela quantidade e diversidade de doenças a ela associadas, como patologias cardiovasculares e cerebrovasculares, distúrbios metabólicos, alguns tipos de câncer, e patologias do aparelho digestivo. No entanto, somam-se aos impactos fisiológicos aqueles de natureza psicossocial, pela estigmatização e discriminação das pessoas que apresentam essa doença. Sendo a obesidade um atributo físico observável, ela é interpretada e influenciada pelo contexto sociocultural que varia de acordo com o período histórico (FERREIRA; MAGALHÃES, 2006). Quanto a esse aspecto, Puhl e Brownell (2001) ressaltam que as pessoas obesas são ridicularizadas publicamente, em escolas, lojas, restaurantes, e, ainda, são alvos de piadas e anedotas.

No Brasil, com prevalência próxima de $60 \%$, o excesso de peso é um dos maiores problemas de saúde (ABESO, 2015). Esse avanço do excesso de peso no Brasil foi constatado mediante dados obtidos pela Pesquisa Nacional sobre Saúde e Nutrição (PNSN). O agravo foi diagnosticado em 27 milhões de indivíduos, correspondendo a 32,0\% da população brasileira total. Desses, 6,8 milhões foram considerados obesos por apresentarem Índice de Massa Corporal (IMC) igual ou superior a $30 \mathrm{~kg} / \mathrm{m} 2$. Os elevados índices de 
obesidade observados nos últimos dez anos alertam para um importante problema de saúde pública que demanda a verificação dos graus de discriminação, preconceito e proteção aos indivíduos obesos (FELIPPE, 2004).

A obesidade se configurou como um fenômeno presente na zona urbana em detrimento da área rural, em todas as regiões brasileiras. Diferenças na prevalência da obesidade entre os grupos sociais também foram observadas. O impacto foi notório na população adulta feminina, especialmente, aquela inserida nos menores percentis de renda. Dos 6,8 milhões de obesos diagnosticados pela PNSN 4, 70,0\% eram do sexo feminino. A população de baixa renda foi a mais atingida, apresentando a prevalência superior a $30,0 \%$ do total de mulheres com excesso de peso, uma proporção considerada preocupante, pois aponta para um sério problema alimentar no Brasil (FERREIRA; MAGALHAES, 2005). A pesquisa do IBGE mais recente aponta que essa situação não só permanece, como também está agravando: um em cada cinco brasileiros de 18 anos ou mais em 2013 (20,8\%) é obeso, sendo o percentual mais alto entre as mulheres $(24,4 \%)$ do que entre os homens(16, 8\%). (ABESO, 2015).

No entanto, o perfil da obesidade apresenta comportamento pouco homogêneo, haja vista que diferenças regionais e entre grupos populacionais são evidenciadas. Por exemplo, em pesquisa realizada no início dos anos 2000, a obesidade mostrou ser mais frequente em áreas consideradas de maior desenvolvimento, tais como o Sul e o Sudeste do país, pois apenas a região Sul totalizava cerca de 5 milhões de adultos com excesso de peso (FERREIRA; MAGALHAES, 2005). Pesquisas apontam, também, a obesidade como um fenômeno urbano nestas regiões, sendo mais acentuada no grupo feminino, mais especificamente, nas mulheres de baixa renda, o que demanda políticas públicas específicas de saúde e nutrição para seu enfrentamento (FERREIRA; MAGALHÃES, 2006). Em termos absolutos, o Sudeste é a região que apresentou, no estudo de Ferreira e Magalhães (2005), a situação mais grave com, aproximadamente, 10 milhões de adultos com sobrepeso e cerca de 3 milhões e meio com obesidade grau 4.

\section{OBESIDADE E ORGANIZAÇÕES}

A temática obesidade e organizações tem fundações interessantes e variadas, ainda que pouco se tenha explorado sobre ela no campo de estudos das organizações e gestão (LEVAY, 2013; LOPES; MEDEIROS, 2015; LEVRINI, 2016). De fato, a temática obesidade tem sido tratada nos campos da saúde (ver PUHL; HEUER, 2009; STEEVES et al., 2012; 
ROMAN et al., 2015, entre outros), da psicologia (ver PUHL; MOSS-RACUSIN; SCHWARTZ, 2007; MORAES; CAREGNATO; SCHNEIDER, 2014; STUNKARD; WADDEN, 1992, entre outros), e da sociologia (ALMEIDA; LOUREIRO; SANTOS, 2002; MONAGHAN, 2010, entre outros) com certa recorrência, porém, sem estabelecer ligações com o contexto das organizações.

Já no campo das organizações, mais especificamente, encontramos contribuições em âmbito internacional, como as de Levay (2013), Schulte et al. (2007), Rothblum (1992) e Witkowski (2007), para destacar algumas. Em âmbito nacional, as pesquisas de Andrade e Chamon (2005) e de Nespeca e Cyrillo (2011) focalizam Qualidade de Vida no Trabalho e sua relação com a obesidade, bem como as de Lopes e Medeiros (2015) e de Levrini (2016).

Levay (2013) argumenta que a obesidade é um tema esquecido que merece ser investigado no campo dos estudos organizacionais (EOs), de modo a acompanhar o recente e crescente interesse na temática por parte de estudiosos. A autora considera que a obesidade desempenha um papel importante na vida organizacional cotidiana, como uma fonte de discriminação, legitimação de diferenças de poder e ansiedade generalizada, mesmo para os não obesos. É nesse sentido que a autora coloca em pauta as implicações da designação da obesidade como uma doença, como, por exemplo, o estabelecimento de IMC como normatividade.

Outro aspecto destacado por Levay (2013) é a obesidade como um fenômeno completamente organizado, pois, cada vez mais, ela é entendida como um problema médico e social, além de alvo de grandes esforços para conter a 'epidemia de obesidade'. Esses esforços incluem iniciativas de saúde no local de trabalho, o que oferece um campo empírico profícuo. Para Levay (2013), uma investigação crítica da obesidade no âmbito das organizações é necessária e possível, e deve, por exemplo, considerar a influência social onipresente de campanhas fracassadas de subjugação e resistência, de preconceito e de reflexão crítica.

As reflexões críticas são sinalizadas por Levay (2013) ao observar que os alertas sobre a obesidade estão se espalhando como uma epidemia e se intensificaram desde a década de 1990, aumentando a preocupação geral quanto a obesidade ser uma séria ameaça à saúde (GARD, 2009, 2011; WRIGHT, 2009). O discurso da obesidade como doença tornou-a uma questão que exige um tratamento não apenas individual, mas para a ação pública, especialmente, na forma de programas e regulamentos de prevenção dirigidas às crianças, famílias e escolas (WRIGHT, 2009). Mais uma vez, o IMC desempenha um papel central 
nesse contexto, por permitir que a obesidade seja convenientemente monitorada e comparada em diferentes populações e ao longo do tempo.

Witkowski (2007) discute a ética do marketing de alimentos no contexto em que a obesidade torna-se uma questão de saúde pública. A discussão do autor gira em torno da ideia de que a indústria de alimentos tornou-se um grande negócio e as empresas do setor devem prover os consumidores de informações nutricionais. Isso porque as mudanças socioculturais, principalmente nos grandes centros urbanos, modificaram os hábitos alimentares, aumentando a demanda e consumo de alimentos altamente calóricos, levando as empresas a oferecer uma variedade de novos produtos.

A relação entre obesidade, saúde e trabalho é o foco dos estudos de Schulte et al. (2007) e de Rothblum (1992). O primeiro oferece cinco modelos conceituais da inter-relação entre trabalho, obesidade e segurança e saúde ocupacional, destacando aspectos éticos, legais e sociais relacionadas à obesidade na saúde e segurança ocupacional. O segundo estudo discute a relação entre obesidade, discriminação no trabalho e vitimização do obeso. $\mathrm{O}$ autor aponta que os obesos relataram sofrer discriminação no emprego e na escola mais do que os indivíduos não obesos. Ainda, os participantes da pesquisa relataram as tentativas de esconder o peso e a queda da autoconfiança, o que ocorre mais entre as mulheres quando comparadas aos homens. Na área da saúde, Steeves et al. (2012) buscam correlações entre a obesidade e o tipo de atividade ocupacional, identificando que as pessoas que ocupam cargos que demandam atividades físicas correm menor risco de obesidade abdominal.

Em âmbito nacional e no contexto das organizações, destacamos a pesquisa de Andrade e Chamon (2005), Nespeca e Cyrillo (2011), Lopes e Medeiros (2015) e Levrini (2016), que relacionam obesidade e Qualidade de Vida no Trabalho (QVT), embora não centralizem o campo organizacional. Os primeiros procuram identificar se o sobrepeso e a obesidade interferem no modo como os funcionários de uma empresa do setor automobilístico veem e agem no ambiente laboral. Os resultados apontam que $73 \%$ dos funcionários com sobrepeso ou obesidade afirmam estarem cansados ao final da jornada de trabalho, o que é entendido pelos autores como uma consequência da obesidade. Nespeca e Cyrillo (2011) também pesquisam essa relação estudando os determinantes da QVT dos funcionários da Universidade de São Paulo, defendendo a hipótese de que o sobrepeso e obesidade teriam efeito negativo sobre a QVT, o que foi confirmado para a amostra de um público jovem predominantemente feminino, com elevada escolaridade, alta incidência de sobrepeso e obesidade. 
Lopes e Medeiros (2015) discutem a manifestação do preconceito contra a obesidade no ambiente de trabalho, pesquisando pessoas obesas e ex-obesas que submeteramse à cirurgia bariátrica. As autoras identificaram que, na interpretação dos pesquisados, a estigmatização do corpo obeso ocorre em virtude das três circunstâncias apontadas por Goffman (1975): a abominação do corpo obeso, a incapacidade do corpo obeso e o corpo obeso associado ao fracasso. Na mesma direção, Levrini (2016) buscou compreender a influência da obesidade nas relações de trabalho e a discriminação contra as pessoas obesas, tendo identificado a condição de estigmatizadora da obesidade, a qual provoca discriminação, exclusão social e preconceito.

A obesidade pesquisada no contexto organizacional demanda olhares diversos, visto serem ambos (obesidade e organizações) campos de natureza multidisciplinar. Para a compreensão da obesidade no contexto das organizações, estudos com abordagens variadas podem oferecer respostas e caminhos cujos desdobramentos podem trazer mudanças concretas para os indivíduos que vivenciam situações em que o preconceito, a discriminação e a estigmatização do obeso se fazem presentes. Assim, na próxima seção, articulamos as conversações de pesquisas sobre a obesidade com o objetivo de oferecer uma visão desse campo profícuo.

\section{QUANTO AO MÉTODO}

Este artigo é de natureza teórica, pois nosso objetivo é oferecer uma agenda para estimular conversações de pesquisa sobre a obesidade na análise organizacional, considerando que esse constitui-se em um tema de extrema relevância, considerando o atual contexto das organizações, o qual é permeado pelas discussões de diversidade e valorização da diferença. Inicialmente, realizamos uma revisão teórica nas principais bases de dados de periódicos nacional e internacional (Spell.org, Portal Capes, Scielo e Sage) e eventos da área de administração, visando identificar estudos que relacionem obesidade e organizações. Em seguida, realizamos um levantamento bibliográfico em bases de periódicos da área de saúde (medicina, educação física e nutrição), psicologia e sociologia. Isso permitiu identificar que a discussão do tema no campo da administração é rara, mas, apesar disso, é um campo promissor, considerando-se as discussões e aproximações realizadas com outros campos de conhecimento.

O próximo passo para encontrar insights que estimulem conversações necessárias sobre obesidade e organizações foi encontrar, dentro do campo da administração, um espaço 
teórico para iniciar essa aproximação, que entendemos ser a discussão da diversidade e das diferenças. Nos estudos em outros campos do conhecimento, a discriminação e o preconceito contra a obesidade são retratados como um dos custos psicossociais que incorrem sobre as pessoas obesas, além daqueles fisiológicos.

Considerando esse espaço, estruturamos nossa análise em dois pontos: (1) o discurso do corpo ideal e (2) o discurso do corpo obeso. A partir desses dois pontos, elaboramos uma matriz que sugere possíveis vias para pesquisas futuras sobre obesidade e organizações em dois eixos: (1) os significados simbólicos da obesidade nos processos ou práticas organizacionais e (2) o corpo como identidade.

\section{Figura 1 - Pesquisas sobre obesidade e organizações}

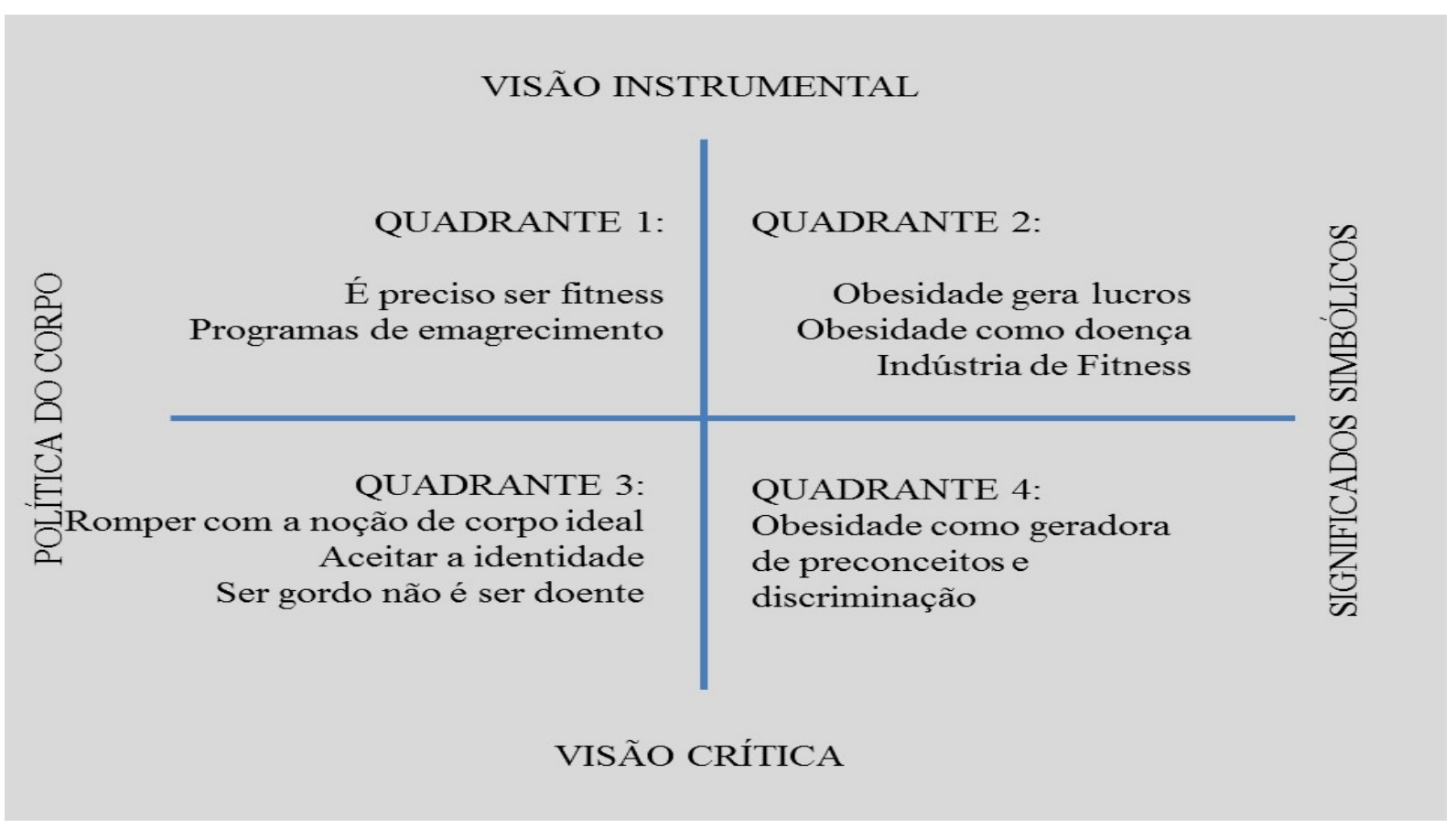

Fonte: elaborada pelos autores.

Consideramos que as conversações propostas não devem privilegiar uma única postura epistemológica, sendo assim, para compor a matriz, consideramos que as pesquisas possam ser realizadas em uma visão instrumental, que se orienta pelo paradigma funcionalista, bem como em uma visão crítica, que se ancora em perspectivas questionadoras da normatividade e do status quo, buscando ir além do que é imposto e dito como ideal.

O primeiro ponto focal, o discurso do corpo ideal, diz respeito aos padrões estabelecidos na sociedade e ao modo pelo qual o corpo determina posições sociais. $\mathrm{O}$ segundo ponto focal, o discurso do corpo obeso, focaliza a estigmatização da obesidade. 
Quanto aos eixos, o primeiro analisa os significados simbólicos da obesidade nos processos organizacionais, repousando nas interpretações atribuídas à obesidade e suas implicações para os processos organizacionais. O segundo eixo analisa o corpo na construção da identidade dos indivíduos.

Para a discussão dos quadrantes produzidos pela matriz, nos inspiramos em duas ideias centrais: o processo de estigmatização e a política do corpo. O processo de estigmatização é discutido a partir de E. Goffman (1975), que conceitua o estigma como a relação entre atributo e estereótipo, cuja origem é ligada à construção social dos significados através das interações sociais. Para a discussão sobre a política do corpo, baseamo-nos no conceito de biopolítica de Foucault (2008), cujo ideia central é de que a sociedade é constituída por corpos, e não por indivíduos. Dessa maneira, o corpo reproduz as relações de poder, sendo balizador de posições sociais, incluindo e excluindo.

\section{CONVERSAÇÕES DE PESQUISA SOBRE OBESIDADE E ORGANIZAÇÕES}

Nesta seção, apresentamos as conversações de pesquisas sobre diversidade nos utilizando de uma matriz, apesar de reconhecer o seu caráter reducionista, de um tema cuja complexidade não pode ser por ela abrangida. No entanto, ao utilizarmos a matriz (Figura 1), buscamos oferecer uma contribuição para os interessados no tema, qual seja, a obesidade no contexto organizacional.

\subsection{QUADRANTE 1: POLÍTICA DO CORPO E VISÃO INSTRUMENTAL}

As questóes centrais desse quadrante são: 'Quais as iniciativas organizacionais orientadas para obter vantagem da política do corpo? E qual sua efetividade?'. As ideias centrais de pesquisas nesse quadrante giram em torno da necessidade de ser fitness, o que se reflete nas iniciativas das organizações relacionadas a programas para emagrecimento visando à saúde do corpo. Essa área de pesquisa é profícua no âmbito das pesquisas em administração, com maior ênfase nas áreas de gestão de pessoas e marketing.

Conhecer e cuidar do corpo é um marco do desenvolvimento de uma economia de consumo de produtos e serviços vinculados a indústrias farmacêuticas, da saúde, editoriais, turísticas, de comunicação, educação, esporte, alimentação, para citar as principais. Assim, a política do corpo saudável e ideal enseja o desenvolvimento de indústrias destinadas a 
produzir belos corpos, oferecendo a possiblidade de esculpir corpos por meios diversos, como procedimentos cirúrgicos.

Ainda, a política do corpo enseja a criação de modelos para gerenciar a força de trabalho diversa. A gestão da diversidade (COX JR.; BLAKE, 1991; FLEURY, 2000) consiste no planejamento de sistemas e práticas organizacionais de gestão de pessoas com a finalidade de maximizar as potenciais vantagens advindas da diversidade e, ao mesmo tempo, minimizar as suas desvantagens. Para Cox Jr. e Blake (1991), gerir a diversidade significa maximizar as habilidades dos trabalhadores de modo que esses contribuam de forma efetiva para o alcance de objetivos da organização, sendo a diversidade uma condição crucial para que as organizações globais alcancem níveis altos de desempenho.

Relacionado a esse quadrante incluem-se estudos que buscam compreender estratégias de marketing social utilizadas para reduzir a obesidade, como a pesquisa de Gracia-Marco et al. (2011). Essa pesquisa apontou que as o marketing social não tem contribuído para as metas de redução de obesidade, visto que as estratégias utilizadas não se mostraram consistentemente utilizadas em intervenções para prevenir obesidade em crianças e adolescentes.

Nesse sentido, as pesquisas que consideram a obesidade e a visão instrumental têm um amplo espectro de possibilidades: (1) pesquisar práticas e programas para a saúde do corpo e redução do peso nas organizações, buscando identificar sua efetividade; (2) estudar comparativamente o desempenho de pessoas com diferentes graus de obesidade, de modo a evidenciar variáveis e fatores que, juntos, podem contribuir para o melhor desempenho; (3) pesquisar o ambiente laboral, mais especificamente, o layout de móveis e espaço, visando a sua adequação para pessoas obesas, sejam elas trabalhadores internos ou consumidores/clientes; (4) investigar os custos associados à obesidade, tanto para o Estado, para a organização e para os indivíduos; e (5) pesquisar o tipo de alimentação oferecido pela organização no ambiente organizacional.

\subsection{QUADRANTE 2: SIGNIFICADOS SIMBÓLICOS DA OBESIDADE E VISÃO INSTRUMENTAL}

Esse quadrante abriga pesquisas que buscam responder questões como 'Quais os significados simbólicos sobre a obesidade e como esses orientam as estratégias da indústria de fitness?'. São pesquisas orientadas para a compreensão dos significados simbólicos em uma visão instrumental, ou seja, a obesidade é vista como geradora de lucros, pois associada 
à doença e contraposta ao corpo ideal e normativo, a obesidade fomenta a indústria de fitness. O corpo obeso ganha na contemporaneidade o significado simbólico de uma doença que deve ser combatida não só com políticas públicas, mas, também, com a oferta de produtos e serviços que prometem um corpo magro e esbelto.

Para Witkowski (2007), a obesidade no mundo dos menos abastados é um problema de saúde muito expressivo para ser ignorado. Com o desenvolvimento econômico, o trabalho manual se tornou menos exigentes fisicamente, rendas discricionárias subiram e os homens e mulheres têm procurado oportunidades nas grandes cidades, onde eles se tornaram menos ativos e tiveram mais oportunidades para comer alimentos ricos em energia. Outro fator que caminha nessa direção é a liberalização econômica desenfreada e a globalização de mercados que permitiram aos comerciantes de alimentos introduzirem uma ampla variedade de novos produtos, distribuí-los de forma abrangente a preços razoáveis, adaptá-los às culturas locais e promovê-los para os públicos-alvo mais sensíveis, especialmente, crianças.

O fato é que, como Witkowski (2007) observa, poucos discordariam da ideia de que a indústria de alimentos mostra mais virtude do que as empresas de tabaco, mas as decisões do mix de marketing dessa indústria, em última instância, podem ter consequências igualmente graves para a saúde de centenas de milhões de consumidores em todo o mundo. Apesar das várias justificações para uma posição de laissez faire inerente à teoria de gestão de marketing, uma análise ética com base nesses mesmos princípios indica que as empresas do setor alimentar têm responsabilidade de informar os consumidores sobre os indicadores de nutrição e saúde dos alimentos. Além disso, essas devem oferecer um número menor de alternativas de alimentos com alta densidade energética a preços mais acessíveis (GRACIAARNAIZ, 2010); porém, isso tem sido feito como uma estratégia de relações públicas (GÓMEZ et al., 2011).

Contribuindo para a criação e disseminação desse significado, a mídia reforça a obesidade como socialmente contranormativa, quer se trate de comédias de situação, desenhos animados, filmes, anúncios ou notícias, podendo até mesmo ser cruel para com as pessoas com sobrepeso (PUHL; HEUER, 2009; MARIANO; MONTEIRO; PAULA, 2013). Ainda, é onipresente a publicidade de produtos e programas de perda de peso que enfatizam a mensagem de que o peso é facilmente modificável e que a perda de peso bem sucedida é uma simples questão de esforço pessoal (FELIPPE, 2004; PUHL e HEUER, 2009).

Kubota (2014) e Felippe (2004) apontam para a contradição existente no mercado de fitness e produtos saudáveis. Para Kubota (2014), por um lado, os padrões de beleza contemporâneos no Ocidente louvam a magreza, especialmente para as mulheres. Por outro, 
o baixo custo de comidas processadas e ricas em gorduras trans e saturadas, xarope de milho rico em frutose, farinhas processadas e outras substâncias contribuem para o ganho de peso. Além disso, o estilo de vida moderno induz a um gasto cada vez menor de calorias por meio de atividades físicas.

No âmbito dessa discussão, Palma et al. (2014) questionam se há possíveis conflitos de interesses na atual 'guerra' contra a obesidade, tendo em vista que fabricantes de alimentos ultraprocessados têm se associado a entidades científicas que desenvolvem ações de estímulo à redução da gordura corporal e ao aumento da prática de atividades físicas. Isso porque, ainda que as pesquisas apontem que a obesidade e o sedentarismo têm crescido no Brasil e no mundo, a guerra contra a obesidade não combate essas condições, mas tem se voltado contra os obesos e sedentários (PUHL; PETERSON; LUEDICKE, 2013; PALMA et al., 2014).

Uma agenda de pesquisa nesse quadrante considera estudos sobre o mercado consumidor de produtos fitness e a criação e desenvolvimento desses produtos: (1) pesquisar estilos de vida de pessoas obesas e não obesas, visando o desenvolvimento de produtos e serviços que considerem as características de diferentes estilos e de diferentes grupos; (2) mapear a indústria de fitness e de produtos/serviços criados com a finalidade de tornar o corpo magro, investigando as estratégias empresariais dessa indústria; (3) investigar o conhecimento dos consumidores acerca das informações dos produtos dessa indústria; (4) pesquisar a opinião dos consumidores quanto à responsabilidade das empresas sobre o crescimento dos índices de obesidade.

\subsection{QUADRANTE 3: POLÍTICA DO CORPO E VISÃO CRÍTICA}

A questão central aqui é: 'De que modo são construídas e desconstruídas as identidades de pessoas obesas?'. O Quadrante 3 compreende pesquisas que considerem a política do corpo e uma visão crítica. Romper com o corpo ideal e aceitar a identidade, questionando, inclusive, as questões associadas à premissa de que ser gordo é ser doente, constitui-se na ideia central de pesquisas nessa área. O estigma e a discriminação contra as pessoas obesas são profundos e representam numerosas consequências para a saúde física e psicológica de quem é obeso.

Puhl e Heuer (2010) observam que, apesar de décadas de ciência que documentam o estigma de peso, as suas implicações para a saúde pública são amplamente ignoradas. Em vez disso, pessoas obesas são culpadas por seu peso, com percepções comuns 
de que a estigmatização de peso é justificável e pode motivar os indivíduos a adotarem comportamentos mais saudáveis. Os autores examinaram evidências para responder a essas premissas e discutir suas implicações na saúde pública e, com base nos resultados, argumentam que o estigma de peso não é uma ferramenta benéfica para reduzir a obesidade, pelo contrário, a estigmatização de indivíduos obesos ameaça a saúde, gera disparidades e interfere nos esforços de intervenção eficazes contra a obesidade. Isso porque, conforme Mariano, Monteiro e Paula (2013), a supervalorização do corpo estético imposto pela sociedade, a discriminação e o preconceito social vivido pelas mulheres obesas favorecem o desenvolvimento da depressão, distúrbios de comportamento e percepção alterada da autoimagem, interferindo com a sua experiência sexual.

Nesse sentido, o corpo ganhou tamanha evidência na sociedade contemporânea que, tal como ocorre com outros estigmas, os estereótipos sobre a pessoa obesa são predominantes e raramente desafiados na sociedade ocidental, deixando as pessoas com sobrepeso e obesas vulneráveis à injustiça social, tratamento injusto e qualidade de vida prejudicada como resultados de desvantagens substanciais e estigma (PUHL; HEUER, 2009). Gomes (2006, p. 68), ao discutir a obesidade como metáfora, ilustra bem o estereótipo do obeso ao observar que esse "parecer ser aquela figura que nos relembra todos os dias que devemos sempre nos esforçar para não ficarmos como ele".

Mariano, Monteiro e Paula (2013) também apontam para a forte tendência sociocultural de considerar a magreza como a situação ideal de aceitação, autocontrole e competência, ainda que a indústria de alimentos esteja em crescimento, ofertando uma variedade de produtos altamente calóricos no mesmo compasso que o número de indivíduos cresce em âmbito mundial.

Além do mais, atitudes negativas podem levar o paciente obeso a evitar a procura de tratamento, como alertam Puhl e Brownell (2001). Segundo os autores, pesquisas relacionam obesidade a uma menor procura por exames pélvicos e ginecológicos, e também associam a obesidade ao número de cancelamento de consultas, o que é atribuído ao embaraço e experiências anteriores negativas de mulheres obesas ao discutirem o seu peso com os médicos.

Ferreira e Magalhães (2005), ancoradas na perspectiva da construção social, afirmam que a obesidade é um atributo físico, percebido, interpretado e influenciado pelo sistema social. Valores socioculturais relacionados à obesidade podem, por conseguinte, variar de uma sociedade para outra, nos diferentes contextos históricos. No entanto, padrões 
definidos para a obesidade assumem contornos distintos em cada sociedade e também entre diferentes grupos sociais.

A valorização do corpo magro e atlético é disseminada e reforçada pela mídia e pela moda com impacto significativo, sobretudo, nas classes média e alta, embora atinja as classes populares, em que se verifica a admiração por modelos e atrizes de televisão que correspondem ao corpo ideal normativo. Segundo Ferreira e Magalhães (2005), contrariamente, existe por parte das camadas socialmente abastadas a vinculação do corpo gordo a pessoas que realizam funções mais modestas, como as cozinheiras, revelando que os estereótipos estão presentes, haja vista que demarcam, muitas vezes, a condição social.

A discriminação contra os obesos ocorre cotidianamente, conforme Puhl e Heuer (2009) e Galassi e Yamashita (2014), seja no trabalho, na escola, na mídia, nos diversos grupos sociais, ou até mesmo na família. Conforme Galassi e Yamashita (2014), na maioria das vezes, os obesos não conseguem expor suas qualidades profissionais, pois são coibidos pelo preconceito social porque a sociedade contemporânea tenta ditar uma regra de padrão físico das pessoas e, para que elas sejam aceitas, precisam se render aos padrões impostos.

O fato é que, conforme Rigo (2012), pelo fato de não ter sido realizado um detalhamento quantitativo específico, a 'nova patologia' não teve uma grande repercussão social. Somente após o estabelecimento de critérios baseados no IMC, em 1998, a condição foi alçada a uma situação em que os sujeitos obesos passaram a ser classificados oficialmente pelas autoridades em saúde como doentes. Na atualidade, a partir de uma classificação das biociências, que colocam a obesidade como doença, o tema tornou-se palco de inúmeras controvérsias éticas e políticas, que vão desde campanhas que reivindicam direitos básicos de cidadania até a denúncia de violência (bullying), preconceito, estigmatização e segregação social (RIGO, 2012).

Nesse quadrante, as pesquisas se orientam para a desconstrução do corpo ideal magro e esbelto como norma social. Sugerimos que as pesquisas nesse quadrante tenham como objetivo: (1) analisar as relações de poder que emergem nas organizações considerando, comparativamente, o corpo obeso e o corpo magro; (2) analisar a mídia gerencialista buscando questionar as representações do corpo ideal no âmbito organizacional; (3) investigar os significados simbólicos do corpo obeso para gestores de pessoas; (4) investigar os processos de exclusão, considerando a interseção da obesidade com gênero, classe social e etnia; e (5) pesquisar profissões e ocupações, como de liderança, que exigem o corpo magro e rejeitam o corpo obeso. As contribuições desse quadrante podem ser fortalecidas com estudos sobre o enfrentamento ao estigma, um fenômeno crescente, 
inclusive, nas mídias sociais, com programas e ações voltadas para a valorização do corpo, independentemente do tamanho. Esse fenômeno é acompanhado pelo crescimento de negócios que ofertam produtos e serviços voltados para pessoas obesas, não com orientação fitness, mas considerando a aceitação de um corpo que foge dos padrões ditados socialmente.

\subsection{QUADRANTE 4: SIGNIFICADOS SIMBÓLICOS DA OBESIDADE E VISÃO CRÍTICA}

O Quadrante 4 abrange estudos que se orientam para responder à questão: 'Como o trabalhador obeso é construído e experienciado nos diferentes contextos organizacionais?'. São pesquisas que focalizam os significados simbólicos da obesidade em uma visão crítica. Aqui a obesidade é geradora de preconceitos e discriminação nas organizações, trazendo implicações para a identidade das pessoas obesas.

Para Levay (2013), a obesidade desempenha um papel importante na vida organizacional cotidiana como uma fonte de discriminação, legitimação de diferenciais de poder e ansiedade generalizada, mesmo para os não obesos. A obesidade também é um fenômeno completamente organizado, sendo cada vez mais entendida como um problema médico e social e alvo de grandes esforços para conter a 'epidemia de obesidade'. Esses esforços compreendem programas e práticas organizacionais com o objetivo de estimular os trabalhadores a se engajarem nos cuidados com o corpo, principalmente, com a redução do peso, o que é apontado, nesse âmbito, como doença.

A tarefa e promessa de estudos críticos da obesidade e organizações é submeter esses processos e práticas a uma crítica sistemática e fazê-lo de uma forma teórica, empírica e politicamente informada (MONAGHAN, 2010). Por exemplo, discutir a obesidade como metáfora para aquilo que deve ser expurgado da vida cotidiana, ou aquilo que é inadequado para uma sociedade em que a beleza tem um significado de excluir e incluir (GOMES, 2006).

Carr e Friedman (2005) oferecem evidências de que pessoas obesas são estigmatizadas e que essa estigmatização transcende avaliações negativas por outros. Segundo os autores, os indivíduos obesos percebem que são alvo de múltiplas formas de discriminação que têm implicações para seu bem-estar psicológico. Nesse entendimento, Santolin e Rigo (2015), considerando a polissemia e a redundância discursiva que ressaltam a obesidade como um mal, consideram que o discurso patológico da obesidade é carregado de valores morais e políticos. Por isso, é necessário evidenciar as relações genealógicas que fizeram com que a obesidade fosse classificada como uma patologia. 
Há uma clara e consistente literatura científica mostrando o preconceito generalizado contra as pessoas com excesso de peso, evidenciando um padrão consistente de discriminação, seja no trabalho, em restaurantes, na escola e em outros espaços públicos. Há de se considerar que as pessoas obesas são alvo frequente de piadas e anedotas, sendo frequentemente ridicularizadas, recebem piores notas na escola, perdem oportunidades em processos de adoção de criança, além de terem promoções e admissões em empregos negadas (PUHL; BROWNELL, 2001).

Puhl e Brownell (2001) evidenciam, no âmbito do trabalho, o preconceito contra obesos na contratação, através dos relatos de entrevistados que afirmam estarem menos inclinados a contratar uma pessoa com sobrepeso do que uma pessoa magra, mesmo com qualificações idênticas. Os entrevistados fazem inferências negativas sobre pessoas obesas no local de trabalho, tais como a suposição de que as pessoas obesas são preguiçosas, indisciplinadas e menos competentes que aquelas não obesas. Desse modo, os autores ponderam que pode-se esperar que essas atribuições afetem os salários, promoções e ações disciplinares, o que parece ser o caso (PUHL; BROWNELL, 2001).

A saúde é outra área em que as atitudes preconceituosas são um problema. Muitas atitudes negativas sobre os indivíduos com excesso de peso têm sido relatadas por médicos, enfermeiros e estudantes de medicina, da mesma forma como na sociedade em geral (PUHL, BROWNELL, 2001). No âmbito organizacional, segundo Puhl e Brownell (2001), muitos obesos relatam mais tipos de discriminação no emprego, opressão escolar, tentativas de esconder o peso e diminuição da autoconfiança do que os sujeitos não obesos. Entre os tipos de discriminação, Rothblum (1992) aponta apelidos negativos, exclusão nos esportes e encontros sociais, e, no caso de professores contra estudantes, humilhações, baixas notas e negativas de cartas de recomendação são recorrentes. Costa, Souza e Oliveira (2012) também estudaram a ocorrência de bullying contra crianças obesas, identificando que essas crianças enfrentam problemas de exclusão, timidez e baixa autoestima, entre outras, além de sofrerem atitudes negativas por parte de professores que, muitas vezes, as descrevem como apáticas, desanimadas, cansadas, lentas, distraídas, preguiçosas, indispostas, entre outras adjetivações.

Conforme Puhl, Moss-Racusin e Schwartz (2007), indivíduos obesos que internalizam estereótipos negativos baseados no peso podem ser particularmente vulneráveis ao impacto negativo do estigma sobre comportamentos alimentares o que, de certa forma, desafia a noção de que o estigma pode motivar indivíduos obesos a se envolverem em esforços para perder peso. $\mathrm{O}$ fato é que as implicações da discriminação e do preconceito para o bem-estar emocional são os diversos sintomas depressivos e a baixa autoestima. 
Nesse sentido, é urgente e necessário desconstruir os preconceitos em torno da obesidade quanto a determinados atributos associados à pessoa obesa, tais como preguiça, incompetência e outros (BAGRICHEVSKY et al., 2007) que deterioram a sua identidade (GOFFMAN, 1975) e se tornam uma barreira para a contratação e promoção do indivíduo no âmbito do trabalho. A agenda de pesquisa sugerida para esse quadrante envolve estudos que questionem os conceitos, práticas e abordagens que reforçam a estigmatização da pessoa obesa no ambiente de trabalho, ou ainda que remetam às figuras que constituem o terreno do discurso sobre o anormal: o monstro humano, o indivíduo a ser corrigido e a criança masturbadora (FOUCAULT, 2002).

Assim, sugerimos a realização de pesquisas que: (1) questionem os programas e ações implementados em organizações com o objetivo de reduzir o peso para tornar os empregados magros; (2) analisem criticamente programas da mídia, filmes, anúncios publicitários, reportagens e outros materiais que disseminam representações negativas das pessoas obesas; (3) investiguem tipos de discriminação e preconceitos contra pessoas obesas no âmbito organizacional; e (4) investiguem as interpretações de trabalhadores, gerentes, clientes e consumidores sobre o papel e o significado da obesidade no contexto organizacional.

\section{CONSIDERAÇÕES FINAIS}

Neste artigo, discutimos aspectos relevantes sobre a obesidade e organizações com o objetivo de oferecer uma agenda para estimular conversações de pesquisa sobre a obesidade na análise organizacional. A partir de pesquisas publicadas na sociologia, na área da saúde, na psicologia e em outras áreas, elaboramos uma matriz para orientar uma agenda de pesquisa sobre a temática visando contribuir para o campo dos estudos de organizações e gestão.

Os quatro quadrantes que compõem a matriz foram norteados pela combinação de dois eixos: (1) a política do corpo e os significados simbólicos da obesidade; e (2) visão crítica e visão instrumental. O Quadrante 1 tem como conceito norteador a política do corpo em uma visão instrumental, e se contrapõe à visão crítica que se estabelece no Quadrante 3. O Quadrante 2 tem como conceito norteador os significados simbólicos da obesidade, em uma visão instrumental, em oposição ao Quadrante 4 que analisa os significados simbólicos em uma visão crítica. 
A matriz elaborada não tem foco em abordagens que possam oferecer alternativas e direções para o entendimento da obesidade como um fenômeno que faz parte das organizações e, portanto, tem implicações para os processos organizacionais e para a relações pessoais que emergem no âmbito organizacional. Além disso, a matriz elaborada permite questionar pressupostos e verdades organizacionais sobre a obesidade. Ressaltamos que as nossas sugestões de pesquisas para cada quadrante constituem-se em ideias estimuladoras para produzir novos insights, não representando essas qualquer tentativa de esgotamento do tema.

Este artigo pretende oferecer contribuições para ampliar o campo de estudos sobre organizações e gestão, chamando atenção para uma temática de importância evidenciada por estatísticas e situações organizacionais que demandam orientação a respeito das implicações produzidas pela obesidade no contexto organizacional. Para que essa temática se desenvolva no campo de estudo das organizações, sugerimos a formação de grupos de pesquisa de caráter multidisciplinar que possam discutir metodologias adequadas para a pesquisa, considerando a sua complexidade. Ainda, para estimular as pesquisas com essa temática, sugerimos iniciativas como a criação de sessões específicas em congressos e chamadas de trabalhos específicas para publicação em periódicos sobre a obesidade e organizações, de modo que o tema ganhe visibilidade na área e as lacunas sejam preenchidas.

As implicações para a área de organizações e gestão residem nas potencialidades de ampliar o conhecimento sobre uma questão contemporânea que impacta as relações de trabalho, bem como as relações de consumo, na medida em que as problemáticas em torno da obesidade estimulam novas associações e ideias para a análise futura. Ao problematizar a obesidade no campo das organizações, estudos bem delineados e que preencham as lacunas apontadas podem indicar cursos de ações na área de gestão de pessoas, no desenvolvimento de novos produtos e serviços e nos processos organizacionais em geral. Por fim, nosso ensaio é um convite à reflexão sobre os pressupostos implícitos quanto à obesidade e o modo como esses estão vinculados à ideia de um corpo político, à estigmatização, aos preconceitos e discriminação. Essas reflexões, por si só, constituem-se em uma implicação relevante para a área.

\section{REFERÊNCIAS}

ABESO. Quase 60\% dos brasileiros estão acima do peso, revela IBGE. 21/08/2015.

Disponível em: http://www.abeso.org.br/noticia/quase-60-dos-brasileiros-estao-acima-dopeso-revela-pesquisa-do-ibge. Acesso em: 15 fev. 2017. 
ALMEIDA, G. A. N. de; LOUREIRO, S. R.; SANTOS, J. E. A Imagem Corporal de Mulheres Morbidamente Obesas Avaliada através do Desenho da Figura Humana.

Psicologia: Reflexão e Crítica, v.15, n. 2, p. 283-292, 2002.

ANDRADE, M. F. M. DE E.; CHAMON, M. Q. DE O. Excesso de peso e qualidade de vida no trabalho. Revista Brasileira de Gestão e Desenvolvimento Organizacional, v.2, n.2, p. $59-75,2005$.

BAGRICHEVSKY, M.; et al. Sedentário 'sem-vergonha', saudável 'responsável'? Problematizando a difusão do 'estilo de vida ativo' no campo sanitário. In: BAGRICHEVSKY, M.; PALMA, A.; ESTEVÃO, A. (Org.). A saúde em debate na Educação Física. Ilhéus: Editus, 2007. v. 3. p. 209-230.

CARR, D.; FRIEDMAN, M. A. Is obesity stigmatizing? Body weight, perceived discrimination, and psychological well-being in the United States. Journal Health Soc Behav. 46, n. 3, p. 244-59, 2005.

COSTA, M. A. P.; SOUZA, M. A. de; OLIVEIRA, V. M. Obesidade infantil e bullying: a ótica dos professores. Educaçao e Pesquisa: Revista da Faculdade de Educação da Universidade de São Paulo, v. 38, n. 3, p. 653-665, 2012.

COX, T. H.; BLAKE, S. Managing cultural diversity: implications for organizational competitiveness. Academy of Management. The Executive, v. 5, n. 3, p. 45-56, 1991.

FELIPPE, F. L. M. Obesidade como um problema social: novas demandas profissionais ao Serviço Social. KATALYSIS, v. 7, n. 2, p. 239-248, 2004.

FERREIRA, V. A.; MAGALHÃES, R. Obesidade e pobreza: o aparente paradoxo. Um estudo com mulheres da Favela da Rocinha, Rio de Janeiro, Brasil. Cadernos de Saúde Pública, v. 21, n. 6, p.1792-1800, 2005.

FERREIRA, V. A.; MAGALHÃES, R. Obesidade no Brasil: tendências atuais. Revista Portuguesa de Saúde Pública, v. 24, n. 2, p. 71-81, 2006.

FLEURY, M. T. L. Gerenciando a diversidade cultural: experiências de empresas brasileiras. Revista de Administração de Empresas, v. 40, n. 3, p. 18-25, jul./set., 2000 FOLHA DE S.PAULO. Professoras dizem ter sido vetadas por obesidade. Cotidiano. 02/02/2011. Disponível em: http://www1.folha.uol.com.br/cotidiano/2011/02/869371professoras-dizem-ter-sido-vetadas-por-obesidade.shtml Acesso em: 20 maio 2015.

FOUCAULT, M. Os anormais: curso no Collège de France. São Paulo: Martins Fontes, 2002

FOUCAULT, M. Nascimento da Biopolítica: Curso no Collège de France (1978-1979). São Paulo: Martins Fontes, 2008. 
GALASSI, A.; YAMASHITA, A. L.V. Obesidade Mórbida, o peso da exclusão. Revista

Científica da Faculdade Dom Bosco de Cornélio Procópio, v. 2, n. 2, p. 1-18, 2014.

GARD, M. Friends, enemies and the cultural politics of critical obesity research. In: WRIGHT J.; HARWOOD V. (Eds). Biopolitics and the 'Obesity Epidemic': Governing Bodies. New York: Routledge, 2009. p. 31-44.

GARD, M. Truth, belief and the cultural politics of obesity scholarship and public health policy. Critical Public Health, v. 21, n. 1, p. 37-48, 2011.

GOFFMAN, E. Estigma: notas sobre a manipulação da identidade deteriorada. Rio de Janeiro: LTC, 1975.

GOMES, I. M. Obesidade como metáfora contemporânea: uma cruzada saudável em nome do consumo e do risco: Movimento, v. 12, n. 3, p. 45-71, 2006.

GÓMEZ, L. et al. Patrocinio de programas de actividad física por parte de la industria de bebidas azucaradas: ¿salud pública o relaciones públicas? Revista de Saúde Pública, v. 45, n. 2, p. 423-427, abr. 2011.

GRACIA-ARNAIZ, M. Fat bodies and thin bodies: Cultural, biomedical and market discourses on obesity. Appetite v. 55, n. 2, p. 219-225, 2010.

GRACIA-MARCO, L.; VICENTE-RODRÍGUEZ, G.; BORYS, J. M.; LE BODO, T.; PETTIGREW, S.; MORENO, L. A. Contribution of social marketing strategies to community-based obesity prevention programmes in children. International Journal of Obesity, v. 35, p. 472-479, 2011.

ISTO É. O peso do preconceito. Comportamento. Edição 2153, 11/02/2011. Disponível em: http://www.istoe.com.br/reportagens/123968_O+PESO+DO+PRECONCEITO Acesso em: 25 mar. 2015.

KUBOTA, L. C. Discriminação contra os estudantes obesos e os muito magros nas escolas brasileiras. IPEA. Texto para discussão 1928. Brasília: IPEA, 2014.

LEVAY, C. Obesity in organizational context. Human Relations, v. 5, p. 1-21, 2013. LEVRINI, L.A Obesidade nas organizações: o preconceito não declarado. Revista Organizações em Contexto, v. 12, n. 24, p. 165-191, 2016.

LOPES, V. R.; MEDEIROS, C. R. O. O peso social da obesidade: a gordofobia na esfera do trabalho. In: Encontro Nacional de Gestão de Pessoas e Relações de Trabalho, 5, 2015, Salvador. Anais... Salvador: ANPAD, 2015.

MARIANO, M.L.L., MONTEIRO, C.S., PAULA, M.A.B. Cirurgia bariátrica: repercussões na vida laboral do obeso. Revista Gaúcha de Enfermagem, v. 34, n. 2, p. 38-45, 2013. 
McKInSEY GLOBAL INSTITUTE. Obesidade já custa ao Brasil $\mathbf{2 , 4 \%}$ do PIB, diz estudo. Publicado em 21/11/2014. Disponível em: http://wsports.com.br/wsports/tag/mckinsey-global-institute/. Acesso em: 20 maio 2015. MONAGHAN, L. F. Discussion piece: a critical take on the obesity debate. Social Theory \& Health v. 3, n. 4, p. 302-314, 2005.

MORAES, J. da M.; CAREGNATO, R.C.A.; SCHNEIDER, D.da S. Qualidade de vida antes e após a cirurgia bariátrica. Acta Paulista de Enfermagem, v. 27, n. 2, p. 157-164, 2014. MORRIS, S. The impact of obesity on employement. Labour Economics, v. 14, p. 413-433, 2007.

NESPECA, M.; CYRILLO, D. C. Qualidade de vida no trabalho de funcionários públicos: papel da nutrição e da qualidade de vida. Acta Scientiarum - Health Sciences, v. 33, n. 2, p. 187-195, 2011

PALMA, A.; FERREIRA, N. T.; VILAÇA, M. M.; ASSIS, M. Conflitos de interesse na "guerra" contra a obesidade: é possível servir a dois senhores? Saúde \& Sociedade, v. 23, n. 4, p. 47-59, 2014.

PUHL, R. M.; HEUER, C. A. The stigma of obesity: a review and update. Obesity Research, v. 17, p. 941-964, 2009.

PUHL, R.; BROWNELL, K. D. Obesity, bias, and discrimination. Obesity Research, v. 9, p. $788-805,2001$.

PUHL, R. M.; MOSS-RACUSIN, C.; SCHWARTZ, M.B. Internalization of weight bias: implications for binge eating and emotional wellbeing. Obesity Research, v. 15, p. 19-23, 2007.

PUHL, R.; PETERSON, J. L.; LUEDICKE, J. Fighting obesity or obese persons?: public perceptions of obesity-related health messages. International Journal of Obesity, v. 37, n. 6, p. 774-782, 2013.

RIGO, L. C.; SANTOLIN, C. B. Combate à obesidade: uma análise da legislação brasileira. Movimento, v. 18, n. 2, p. 279-296, 2012.

ROMAN, E.P.; RIBEIRO, R. R.; GUERRA-JUNIOR, G.; BARROS-FILHO, A.de A. Comparação do estado nutricional de meninas de acordo com diferentes referências para índice de massa corporal. Revista Brasileira de Saúde Materno Infantil. v. 15, n. 1, p. 121$129,2015$.

ROTHBLUM, E. D. The stigma of women's weight: social and economic realities.

Feminism \& Psychology, v. 2, n. 1, p. 61-73, 1992. 
SANTOLIN, C. B.; RIGO, L. C. O Nascimento do discurso patologizante da obesidade.

Movimento, v. 21, n. 1, p. 81-94, 2015.

SCHULTE, P. A. et al. Work, obesity and occupational safety and health. The American

Journal of Public Health, v. 97, n. 3, p. 428-436, 2007.

STEEVES, J. A.; BASSETT, D. R.; THOMPSON, D. L.; FITZHUGH, E. C. Relationships of occupational and non-occupational physical activity to abdominal obesity. International

Journal of Obesity, v. 36, p. 100-106, 2012.

STUNKARD, A. J.; WADDEN, T.A. Psychological aspects of severe obesity. The

American Journal of Clinical Nutrition. v. 55, n. 2, p. 524-532, 1992.

WORLD HEALTH ORGANIZATION. (WHO) Obesity and overweight: 2014. Disponível em: http://www.who.int/mediacentre/factsheets/fs311/en/. Acesso em: 30 mar. 2015.

WITKOWSKI, T. H. Food marketing and obesity in developing countries: analysis, ethics, and public policy. Journal of Macromarketing, v. 27, n. 2, p. 126-137, 2007.

WRIGHT, J. Biopower, biopedagogies and the obesity epidemic. In: WRIGHT J.; HARWOOD V. (Eds). Biopolitics and the 'obesity epidemic': governing bodies. New York: Routledge, 2009. p. 1-14. 